\title{
Deep Net Model for Detection of Covid-19 using Radiographs based on ROC Analysis
}

\author{
Dr. R. Dhaya \\ Professor, \\ Department of Computer science and Engineering \\ King Khalid University \\ Kingdom of Saudi Arabia \\ dhayavel2005@gmail.com
}

\begin{abstract}
There is a rapid spread of the novel corona virus (Covid-19) among millions of people and causing the death of hundreds of thousands of people according to the analytical data provided by the European Centre for Disease Prevention and Control. However, the number of test kits available for Covid-19 is still limited despite the continuously increasing cases every day. Implementation of an automatic detection system is essential for diagnosis and prevention of the spread of Covid-19. Chest X-ray radiographs are used for the detection of Corona Virus using three significant models of convolution neural network namely Inception- ResNetV2, InceptionV3 and ResNet50. Among the existing systems, the highest performance and classification accuracy is provided by the ResNet50 model. A novel framework based on CNN model is proposed that offers improved specificity, sensitivity and accuracy when compared to the existing models. Fivefold cross validation is used for analysis of the existing models and comparison of the proposed model by means of confusion matrices and ROC analysis.
\end{abstract}

Keywords: Deep Transfer Learning; Convolutional Neural Network; Chest X-ray Radiographs; Pneumonia; Corona Virus;

\section{Introduction}

Since December 2019, the novel corona virus (Covid-19) pandemic has been shaking the earth and has evolved as a serious problem across the planet affecting public health [1]. Severe acute respiratory syndrome coronavirus 2 (SARS-CoV-2) is another name for the Covid-19 pandemic disease causing virus. The Severe Acute Respiratory Syndrome (SARS-CoV) and Middle East Respiratory Syndrome (MERS-CoV) are some of the diseases that belong to the family of Corona viruses $(\mathrm{CoV})$ that result from cold. This species of Corona virus has not been identified in humans previously and is discovered in the year 2019. [2] The contamination from animals to human beings has led to the zoonotic nature of Corona viruses. Dromedary (Somali Camel) contamination is the source of MERS-CoV virus and musk cats are the source of contamination of the SARS-CoV virus according to previous studies and researches [3]. There is a presumption that the Covid-19 virus may be contaminated to humans from bats. There is a rapid spread of this disease through respiratory transmission from person to person.

The symptoms of Covid-19 is critical or sever in about $20 \%$ of the cases and milder in the rest of the cases [4]. During mid-June 2020, the total number of confirmed Covid-19 cases is 7.15 millions of which 408 thousands of them died and over 2 million recovered. This sums up to 1,014 positive cases and 58 deaths for every one million people globally [5]. 5\% of the infected patients are in a critical or serious condition whereas the rest are diseased slightly and can survive the condition. Dyspnea, cough, fever and respiratory infection are some of the symptoms of this disease [6]. Multi-organ failure, septic shock, severe acute respiratory syndrome, pneumonia and even death may occur in serious cases due to the infection. Certain observations show that the rate of infection in men is more than that of women and there is a nil death rate in children of age 0-9. When compared to healthy people, the rate of spread of covid-19 is faster in people affected by pneumonia [7]. Due to the increasing intensity of the disease and the demand for intensive care units, the health systems are facing shortage of resources even in 
Journal of Innovative Image Processing (JIIP) (2020)

Vol.02/ No. 03

Pages: $135-140$

https://www.irojournals.com/iroiip/

DOI:_https://doi.org/10.36548/jiip.2020.3.003

developed countries. Figure 1 represents the Covid-19 highly infected countries over a period of four days with respect to new cases per day.

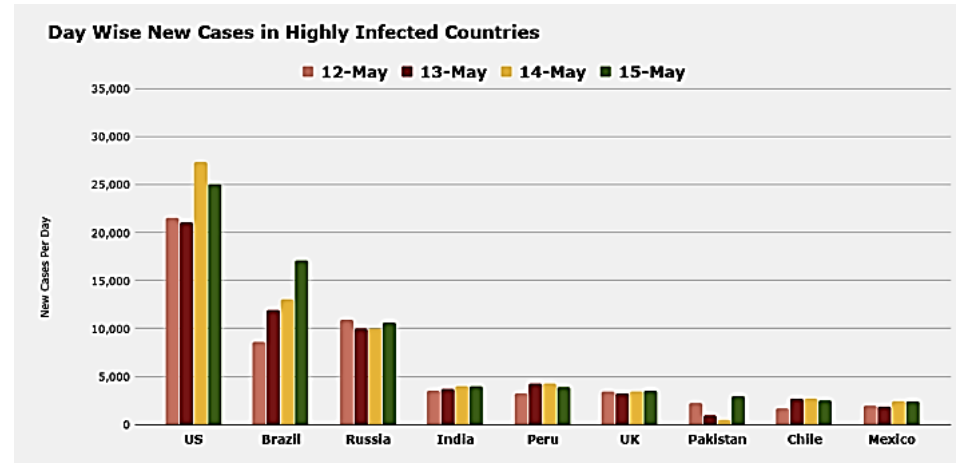

Figure 1: Worldwide distribution of Covid-19 cases [10].

\section{Materials and Methods}

\subsection{Dataset}

For testing, training and analysis of the existing CNN models, we use the repository of chest X-ray images from various relevant databases in GitHub and gathered 50 chest X-ray images of patients with Covid-19 [8]. The database consists of patients with severe acute respiratory syndrome (SARS), pneumonia, Middle East respiratory syndrome (MERS), acute respiratory distress syndrome (ARDS) as well as corona virus disease. Along with this, fifty normal X-ray images of the chest region are also included for comparison [9]. A total of 100 images are used for experimentation and training of the models. Resizing of all images are performed to a pixel size of $224 \times 224$. Figure 2 provides sample X-ray images of the chest region for a normal person and a patient affected by Covid-19 [10].

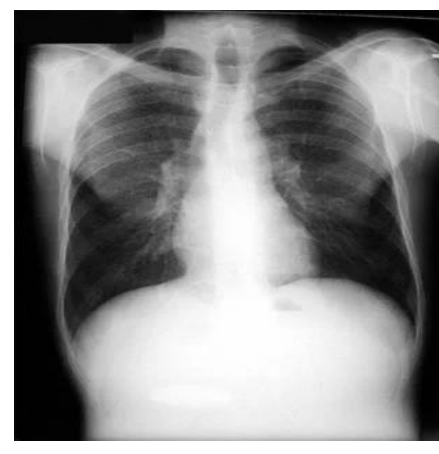

(a)

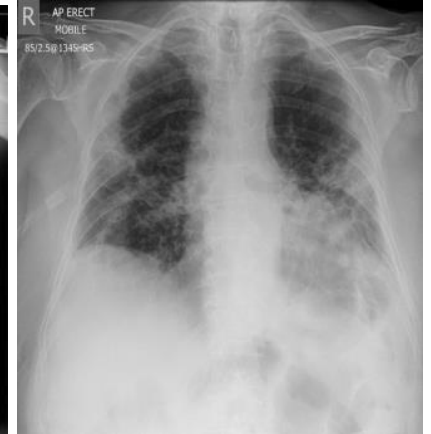

(b)

Figure 2: Representative chest X-ray images of (a) normal and (b) Covid-19 patients 
Vol.02/ No. 03

Pages: $135-140$

https://www.irojournals.com/iroiip/

DOI:_https://doi.org/10.36548/jiip.2020.3.003

\section{Proposed Covid-Caps}

Figure 3 represents the basic architecture of the proposed Covid Capsule model with two layers of capsule network and four layers of convolution. The X-ray images of 3D network are provided as inputs to the system [11]. The batch normalization process follows the first convolutional later. Average polling follows the second convolutional layer. The Capsule network layer follows the third and fourth convolutional layers. The capsule layer is the reshaped fourth layer of convolution. The capsule layer follows an agreement process by routing and two such layers are embedded to form the Covid capsule model [12]. The positive and negative Covid19 parameters are the two classes for instantiation of the capsule network. The length and probability of the classes present are represented by the capsules [13].

As data augmentation is not performed in the proposed architecture based on capsule network, large datasets are not required [14]. Compared to the number of negative cases $\mathrm{N}^{\mathrm{n}}$, the number of positive cases $\mathrm{N}^{\mathrm{p}}$ are lesser and the class imbalance issue is handed by a modified loss function [15]. Determination of the weights for the negative and positive samples with respect to the loss function is performed using the following expression:

$$
\operatorname{loss}=\frac{N^{p}}{N^{p}+N^{n}} \times \operatorname{loss}^{n}+\frac{N^{n}}{N^{p}+N^{n}} \times \operatorname{loss}^{p}
$$

Here, the loss associated with positive samples are represented by $\operatorname{loss}^{\mathrm{p}}$ and the loss associated with negative samples are denoted by loss ${ }^{\mathrm{n}}$.
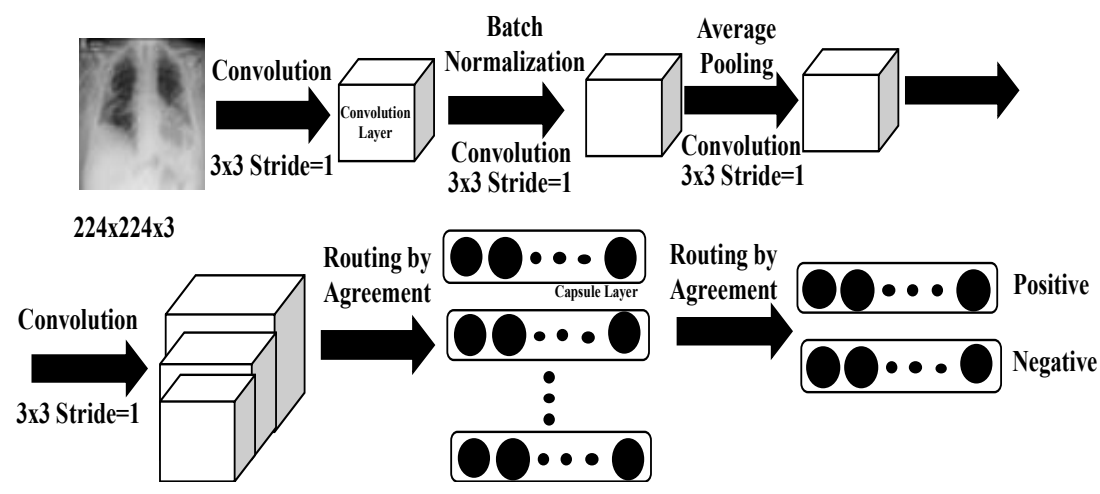

Figure 3: Architecture of the proposed Covid Capsule Model

\section{Results and Discussion}

The patients affected by Corona virus disease are predicted based on the X-ray images of the chest region in this paper. Training and testing of popular CNN models such as Inception- ResNetV2, InceptionV3 and ResNet50 are used for training the X-ray images of the chest region. These pre-trained models are compared for the fold-3 loss values and training accuracy as represented in Figure 4 and Figure 5. 50 epochs of training stages are carried out for all the three above mentioned models. From the graphs, it is visible that ResNet50 model offers the highest accuracy of training. The performance of Inception-ResNetV2 and Inception V3 models are similar. 
Journal of Innovative Image Processing (JIIP) (2020)

Vol.02/ No. 03

Pages: $135-140$

https://www.irojournals.com/iroiip/

DOI:_https://doi.org/10.36548/jiip.2020.3.003

The training speed of ResNet50 is also faster when compared to the other models. Due to limited amount of training data used in this scenario, the initial values are less than $75 \%$ despite high initial values given generally by the pre-trained models. The training loss of Inception- ResNetV2, InceptionV3 and ResNet50 are also compared for the analysis of loss figure. With the increase in the number of training stages for each of the three pre-trained models, the loss values decreases. This decrease appears faster in ResNet50 model and reaches zero based on the experimental results.

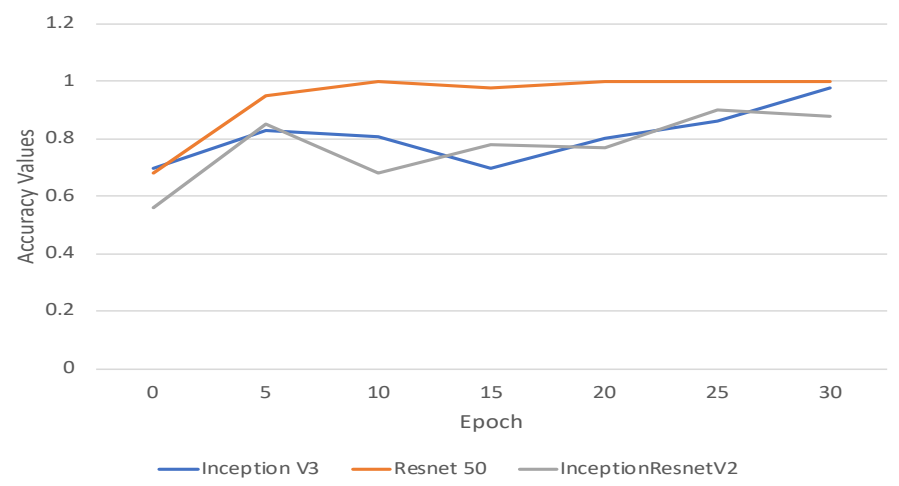

Figure 4: Estimation of performance based on accuracy values of three pre-trained models

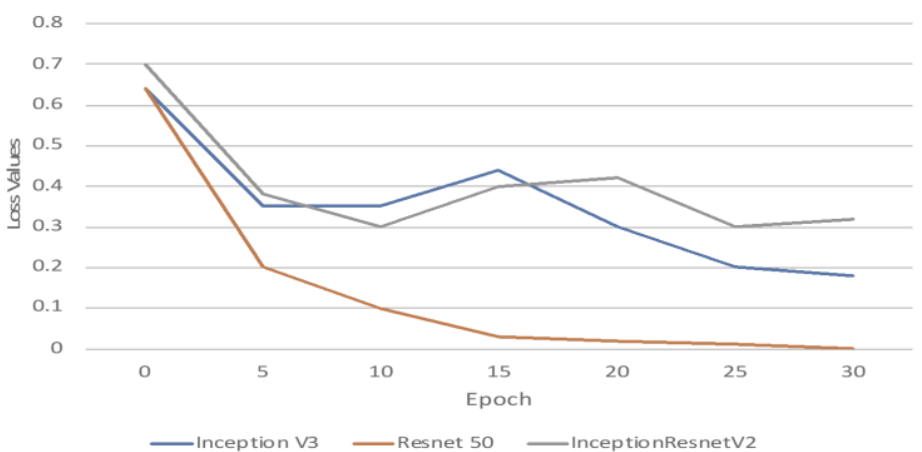

Figure 5: Performance estimation based on training loss for three pre-trained models

The model's normal test results and confusion matrices are analyzed for Covid-19. On implementation of a pretrained model of InceptionV3, the number of true positive out of a set of 50 samples is predicted as 25 and the number of normal or true negative cases is classified as 25. On implementation of the ResNet50 model, similar results as that on InceptionV3 model are obtained. However, the Inception-ResNetV2 classified 24 true negative or normal people and 25 true positive cases. The receiver operating characteristic curve (ROC) plots as well as the confusion matrix for each model is studied. The pre-trained models ResNet50 and InceptionV3 offers higher values. 
Journal of Innovative Image Processing (JIIP) (2020)

Vol.02/ No. 03

Pages: $135-140$

https://www.irojournals.com/iroiip/

DOI:_https://doi.org/10.36548/jiip.2020.3.003

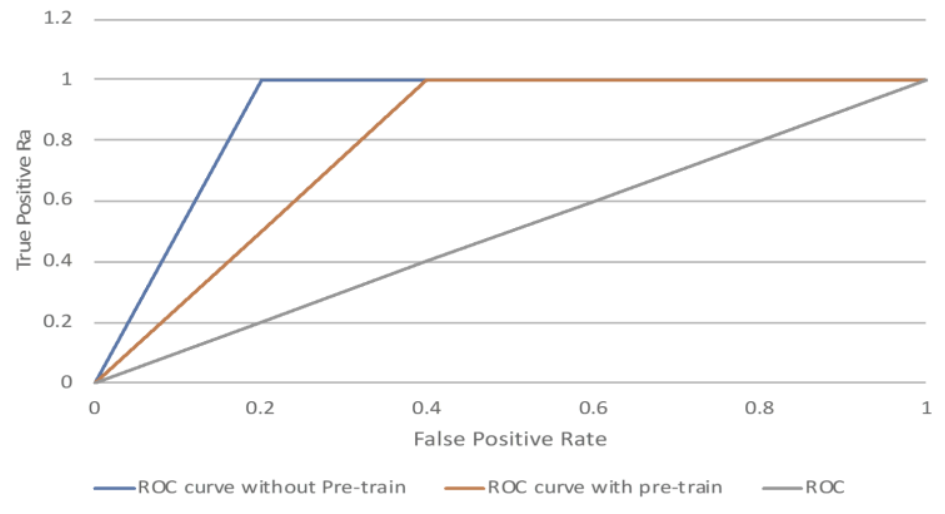

Figure 6: Estimation of ROC curve of the proposed Covid Capsule Model

X-ray image based external dataset with limited number of images is used for pre-training of the proposed Covid-Capsule Model. Multiple datasets are used for the purpose of training and testing the system for estimation of the efficiency. Two more datasets, one consisting of 100 X-ray images and another consisting of 300 thorax disease based X-ray images of the chest region are also used for pre-training the proposed model. The X-ray datasets are obtained from online research and hospital resources. These datasets are inclusive of normal cases in which there are no specific findings corresponding to the images. For reducing the complexity of the system, the images that are extracted are categorized into five groups- one with no symptoms of disease, one with lung infections and the others with pleural diseases, tumors and uncertain images. The uncertain images are those that do not fit into the first four categories and requires further investigation. Images that appear in more than one category are also removed in order to reduce the complexity of the system and for optimization of pre-training. The final dataset obtained after classification in used for pre-training the model with multiple iterations. It is observed that the specificity and accuracy of the model is improved with the number of iterations and fine-tuning of the proposed Covid capsule model based on the results of experimentation. The ROC curve obtained also represents the area under curve that outperforms the existing CNN models.

\section{Conclusion}

In order to prevent the Covid-19 disease spread among people, it is essential to predict the infection at an early stage. For this purpose, we use the X-ray images of the chest region gathered from the patients infected by Covid-19 as well as the normal people for training a system based on deep transfer learning approach. Three CNN models are compared namely Inception- ResNetV2, InceptionV3 and ResNet50 of which ResNet50 provides the highest performance and accuracy of about $98 \%$. The class imbalance problem is overcome using a modified lost function and several layers of convolution and capsule in the proposed framework. Due to the improved performance, decision making for doctors and those in clinical practice will be easier on implementation of this system. The area under curve, specificity and accuracy of the model can be further strengthened by pre-training. The image quantity in the datasets can be increased for improving the performance of model in terms of classification and testing in the subsequent studies. 
Journal of Innovative Image Processing (JIIP) (2020)

Vol.02/ No. 03

Pages: $135-140$

https://www.irojournals.com/iroiip/

DOI:_https://doi.org/10.36548/jiip.2020.3.003

\section{References:}

[1] Apostolopoulos, I. D., \& Mpesiana, T. A. (2020). Covid-19: automatic detection from x-ray images utilizing transfer learning with convolutional neural networks. Physical and Engineering Sciences in Medicine, 1.

[2] Hemdan, E. E. D., Shouman, M. A., \& Karar, M. E. (2020). Covidx-net: A framework of deep learning classifiers to diagnose covid-19 in X-ray images. arXiv preprint arXiv:2003.11055.

[3] Zhang, J., Xie, Y., Li, Y., Shen, C., \& Xia, Y. (2020). Covid-19 screening on chest X-ray images using deep learning based anomaly detection. arXiv preprint arXiv:2003.12338.

[4] Abbas, A., Abdelsamea, M. M., \& Gaber, M. M. (2020). Classification of COVID-19 in chest X-ray images using DeTraC deep convolutional neural network. arXiv preprint arXiv:2003.13815.

[5] Ruth Anita Shirley D, Ranjani K, Gokulalakshmi Arunachalam, Janeera D.A., "Distributed Gardening System Using Object Recognition and Visual Servoing" In International Conference on Inventive Communication and Computational Technologies [ICICCT 2020], Springer, India, 2020.

[6] Hall, L. O., Paul, R., Goldgof, D. B., \& Goldgof, G. M. (2020). Finding covid-19 from chest x-rays using deep learning on a small dataset. arXiv preprint arXiv:2004.02060.

[7] Jin, C., Chen, W., Cao, Y., Xu, Z., Zhang, X., Deng, L., ... \& Feng, J. (2020). Development and Evaluation of an AI System for COVID-19 Diagnosis. medRxiv.

[8] Jayashree, S. and D. A. Janeera. "Real-Time Fire Detection, Alerting and Suppression System using Live Video Surveillance." (2016).

[9] Gozes, O., Frid-Adar, M., Greenspan, H., Browning, P. D., Zhang, H., Ji, W., ... \& Siegel, E. (2020). Rapid ai development cycle for the coronavirus (covid-19) pandemic: Initial results for automated detection \& patient monitoring using deep learning ct image analysis. arXiv preprint arXiv:2003.05037.

[10]D. A. Janeera and Sasipriya.S. "A Brain Computer Interface Based Patient Observation and Indoor Locating System with Capsule Network Algorithm" In International Conference on Image Processing and Capsule Networks (ICIPCN 2020), Springer, Thailand, 2020.

[11] Maghdid, H. S., Asaad, A. T., Ghafoor, K. Z., Sadiq, A. S., \& Khan, M. K. (2020). Diagnosing COVID19 pneumonia from X-ray and CT images using deep learning and transfer learning algorithms. arXiv preprint arXiv:2004.00038.

[12] Khalifa, N. E. M., Taha, M. H. N., Hassanien, A. E., \& Elghamrawy, S. (2020). Detection of coronavirus (COVID-19) associated pneumonia based on generative adversarial networks and a fine-tuned deep transfer learning model using chest X-ray dataset. arXiv preprint arXiv:2004.01184.

[13]Luz, E., Silva, P. L., Silva, R., \& Moreira, G. (2020). Towards an efficient deep learning model for covid19 patterns detection in X-ray images. arXiv preprint arXiv:2004.05717.

[14] Jacob, I. J. (2019). CAPSULE NETWORK BASED BIOMETRIC RECOGNITION SYSTEM. Journal of Artificial Intelligence, 1(02), 83-94.

[15]Bashar, A. (2019). SURVEY ON EVOLVING DEEP LEARNING NEURAL NETWORK ARCHITECTURES. Journal of Artificial Intelligence, 1(02), 73-82.

\section{Authors Biography}

Dr. R. Dhaya is currently Professor, in Department of Computer science and Engineering at King Khalid University Kingdom of Saudi Arabia his research area of interest are Perceptual Modeling and Spectral Imaging Systems, Real time Image Processing, Computer Vision and Pattern Recognition, Image Processing Systems and algorithms for Medical, Industrial, Embedded and Vision assisted Intelligent Robotic Systems, video communication, biomedical imaging, electronic imaging, image and video systems, and remote sensing 